\title{
Correction to: Depressive symptoms in individuals with family members requiring ADL assistance
}

\author{
Junhyun Kwon ${ }^{1,2}$, Eun-Cheol Park ${ }^{2,3}$, Woorim Kim ${ }^{1,2,4}$, Dong-Woo Choi ${ }^{1,2}$ and Sung-In Jang ${ }^{2,3^{*}}$
}

\section{Correction to: Environ Health Prev Med (2019) 24:49 \\ https://doi.org/10.1186/s12199-019-0804-x}

Following publication of the original article [1], the authors reported an error in Table 2 in their paper. The table entry "Men's P value" was mistakenly included under the table heading "Women". The original article [1] has been updated.

The correct Table 2 is shown below.

\section{Author details}

'Department of Public Health, Graduate School, Yonsei University, Seoul,

Republic of Korea. ${ }^{2}$ Institute of Health Services Research, Yonsei University, Seoul, Republic of Korea. ${ }^{3}$ Department of Preventive Medicine, Yonsei

University College of Medicine, 50 Yonsei-ro, Seodaemun-gu, Seoul 120-752, Republic of Korea. ${ }^{4}$ Department of Preventive Medicine, College of Medicine,

Korea University, Seoul, Republic of Korea.

Published online: 28 August 2019

\section{Reference}

1. Kwon, et al. Depressive symptoms in individuals with family members requiring ADL assistance. Environ Health Prev Med. 2019;24:49.

https://doi.org/10.1186/s12199-019-0804-x.

\footnotetext{
* Correspondence: jangsi@yuhs.ac

${ }^{2}$ Institute of Health Services Research, Yonsei University, Seoul, Republic of Korea

${ }^{3}$ Department of Preventive Medicine, Yonsei University College of Medicine,

50 Yonsei-ro, Seodaemun-gu, Seoul 120-752, Republic of Korea

Full list of author information is available at the end of the article
}

(c) The Author(s). 2019 Open Access This article is distributed under the terms of the Creative Commons Attribution 4.0 International License (http://creativecommons.org/licenses/by/4.0/), which permits unrestricted use, distribution, and reproduction in any medium, provided you give appropriate credit to the original author(s) and the source, provide a link to the Creative Commons license, and indicate if changes were made. The Creative Commons Public Domain Dedication waiver (http://creativecommons.org/publicdomain/zero/1.0/) applies to the data made available in this article, unless otherwise stated. 
Table 2 Results of analysis of factors associated with CES-D 10 scores

\begin{tabular}{|c|c|c|c|c|c|c|}
\hline \multirow[t]{2}{*}{ Variables } & \multicolumn{3}{|l|}{ Men } & \multicolumn{3}{|l|}{ Women } \\
\hline & $\beta$ & S.E & $P$-value & $\beta$ & S.E & $P$-value \\
\hline \multicolumn{7}{|c|}{ Caregiving Status of family members requiring $A D L$ assistance } \\
\hline Yes, by others ${ }^{a}$ & 0.035 & 0.089 & 0.6947 & 0.210 & 0.084 & 0.0123 \\
\hline Yes, by myself & 0.407 & 0.145 & 0.0051 & 0.506 & 0.125 & $<.0001$ \\
\hline No family members requiring $A D L$ assistance & Ref. & & & Ref. & & \\
\hline \multicolumn{7}{|l|}{ Age } \\
\hline $45-54$ & Ref. & & & Ref. & & \\
\hline $55-64$ & -0.003 & 0.053 & 0.9488 & 0.089 & 0.052 & 0.0836 \\
\hline $65-74$ & 0.051 & 0.071 & 0.4769 & 0.226 & 0.069 & 0.0010 \\
\hline$\geq 75$ & 0.122 & 0.090 & 0.1742 & 0.326 & 0.082 & $<.0001$ \\
\hline \multicolumn{7}{|l|}{ Education level } \\
\hline Elementary school or less & 0.460 & 0.086 & $<.0001$ & 0.364 & 0.114 & 0.0014 \\
\hline Middle school & 0.272 & 0.090 & 0.0025 & 0.149 & 0.117 & 0.2032 \\
\hline High school & 0.164 & 0.070 & 0.0194 & -0.041 & 0.106 & 0.7000 \\
\hline University or beyond & Ref. & & & Ref. & & \\
\hline \multicolumn{7}{|l|}{ Region } \\
\hline Metropolitan & Ref. & & & Ref. & & \\
\hline Rural & 0.168 & 0.053 & 0.0017 & 0.170 & 0.050 & 0.0007 \\
\hline \multicolumn{7}{|l|}{ Working Status } \\
\hline Working & Ref. & & & Ref. & & \\
\hline Non-working & 0.469 & 0.054 & $<.0001$ & 0.255 & 0.047 & $<.0001$ \\
\hline \multicolumn{7}{|l|}{ Equalized household income } \\
\hline Low & Ref. & & & Ref. & & \\
\hline Mid-low & -0.139 & 0.061 & 0.0213 & -0.321 & 0.054 & $<.0001$ \\
\hline Mid-high & -0.164 & 0.066 & 0.0134 & -0.467 & 0.058 & $<.0001$ \\
\hline High & -0.175 & 0.075 & 0.0194 & -0.411 & 0.066 & $<.0001$ \\
\hline \multicolumn{7}{|l|}{ Perceive health status } \\
\hline Healthy & -1.540 & 0.069 & $<.0001$ & -1.486 & 0.058 & $<.0001$ \\
\hline Average & -1.247 & 0.066 & $<.0001$ & -1.158 & 0.053 & $<.0001$ \\
\hline Unhealthy & Ref. & & & Ref. & & \\
\hline \multicolumn{7}{|l|}{ Regular physical activities } \\
\hline Yes & -0.282 & 0.041 & $<.0001$ & -0.260 & 0.039 & $<.0001$ \\
\hline No & Ref. & & & Ref. & & \\
\hline \multicolumn{7}{|l|}{ Participation in social activities } \\
\hline Yes & -0.407 & 0.056 & $<.0001$ & -0.155 & 0.046 & 0.0008 \\
\hline No & Ref. & & & Ref. & & \\
\hline \multicolumn{7}{|l|}{ Smoking } \\
\hline Ever & 0.021 & 0.056 & 0.7097 & 0.663 & 0.142 & $<.0001$ \\
\hline Never & Ref. & & & Ref. & & \\
\hline \multicolumn{7}{|l|}{ Alcohol intake } \\
\hline Yes & -0.222 & 0.051 & $<.0001$ & -0.118 & 0.059 & 0.0454 \\
\hline No & Ref. & & & Ref. & & \\
\hline \multicolumn{7}{|l|}{ Number of chronic diseases } \\
\hline None & Ref. & & & Ref. & & \\
\hline
\end{tabular}


Table 2 Results of analysis of factors associated with CES-D 10 scores (Continued)

\begin{tabular}{|c|c|c|c|c|c|c|}
\hline \multirow[t]{2}{*}{ Variables } & \multicolumn{3}{|l|}{ Men } & \multicolumn{3}{|c|}{ Women } \\
\hline & $\beta$ & S.E & $P$-value & $\bar{\beta}$ & S.E & $P$-value \\
\hline 1 & 0.007 & 0.055 & 0.8963 & 0.188 & 0.055 & 0.0006 \\
\hline$\geq 2$ & 0.176 & 0.072 & 0.0146 & 0.430 & 0.066 & $<.0001$ \\
\hline \multicolumn{7}{|c|}{ Number of cohabiting generations } \\
\hline Couple & -0.009 & 0.082 & 0.9101 & -0.179 & 0.074 & 0.0154 \\
\hline Two generations & 0.057 & 0.080 & 0.4767 & -0.018 & 0.073 & 0.8022 \\
\hline Over two generations & \multicolumn{3}{|l|}{ Ref. } & \multicolumn{3}{|l|}{ Ref. } \\
\hline
\end{tabular}

ather caregivers include other family members acting as informal caregivers as well as formal/professional individuals 